\title{
Selected extended papers of NFM 2018
}

\author{
Aaron Dutle ${ }^{1} \cdot$ César Muñoz $^{1} \cdot$ Anthony Narkawicz $^{2}$
}

Published online: 16 August 2019

(c) Springer-Verlag London Ltd., part of Springer Nature 2019

This special issue contains extended versions of selected contributions of the 10th NASA Formal Methods Symposium (NFM 2018). The symposium was held on April 17-19, 2018 in Newport News, VA and its proceedings appeared as volume 10811 of the series Lecture Notes in Computer Science (LNCS), published by Springer. The NASA Formal Methods (NFM) Symposium is a forum to foster collaboration between theoreticians and practitioners from NASA, academia, and industry, with the goal of identifying challenges and providing solutions to achieving assurance in mission- and safety-critical systems. The papers appearing in this special issue were carefully reviewed by specialists, including members of the NFM 2018 Program Committee and additional experts. The reviewers guaranteed both significant additional contributions with respect to the LNCS proceedings and assured the quality standards of the Innovation in Systems and Software Engineering a NASA Journal. In the following, a short introduction is given to each of the nine papers included in this volume.

In Formal Modeling and Analysis of Safety-Critical Human Multitasking, G. Broccia, P. Milazzo, and P. Ölveczky define in Real-Time Maude an executable formal model of human attention and multitasking. This formalization supports the analysis of cognitive overload in safety-critical applications using simulation and model-checking.

In On Rewriting Towards Trace Coverage of Symmetric Systems, F. de Paula, A. Haran, and B. Bingham present a framework that combines rewriting and abstraction to construct a coverage model of systems of parameterized symmetric models. This coverage model is used to identify coverage-holes in large systems.

In Model-Based Testing of Stochastically Timed Systems, M. Gerhold, A. Hartmanns, and M. Stoelinga propose model-

$凶$ Aaron Dutle

aaron.m.dutle@nasa.gov

César Muñoz

cesar.a.munoz@nasa.gov

1 NASA, Hampton, VA, USA

2 Hampton, VA, USA based testing frameworks for stochastically timed systems. The frameworks, which are based on Input/Output Markov Automata and Stochastic Automata, cover automatic test case generation, execution and evaluation.

In Optimal Compression of Combinatorial State Spaces, A. Laarman presents a new data structure, namely Compact Tree, for state storage in explicit state model checking. The paper shows that assuming that the state space has a particular format and that it exhibits a few properties related to the transitions of the system, the Compact Tree format is able to reach the information theoretical lower bound for data compression.

In Certified Normalization of Generalized Traces, H. Maarand and T. Uustalu describe generalizations of the Foata and lexicographic normalization procedures for Mazurkiewicz traces, which are used to model concurrent behaviors. These generalizations are formalized in the theorem prover Agda.

In Sound Black-Box Checking in the LearnLib, J. Meijer and J. van de Pol propose improvements to Black Box Checking (BBC) architecture. The improvements are implemented in the LearnLib and evaluated on a collection of the Rigurous Examination of Reactive Systems (RERS) challenge problems.

In Model-checking Task Parallel Programs for Data-race R. Nakade, E. Mercer, P. Aldous, K. Storey, B. Ogles, J. Hooker, S. Powell, and J. McCarthy develop a new algorithmic approach to data-race detection of concurrent programs. The new approach is implemented for Habanero Java, which is integrated to NASA's Java Path Finder (JPF) framework.

In Property Specification Patterns at Work: Verification and Inconsistency Explanation, M. Narizzano, L. Pulina, A. Tacchella, and S. Vuotto present a novel technique to analyze the consistency of system-requirement documents. The paper discusses experimental results on the scalability of the technique and illustrates the proposed technique on a real-life example.

In A Synergistic Approach to Improving Symbolic Execution Using Test Ranges, G. Yang, R. Qiu, S. Khurshid, C. Păsăreanu, and J. Wen introduce the concepts of feasible ranges and unexplored ranges. These concepts aim at achiev- 
ing efficient work distribution for symbolic execution and an efficient reuse of testing results created by other tools.

Last but not least, we wish to thank the Editor-in-Chief, Michael Hinchey, and the editorial office of Springer for their collaborationin producing this special issue, the reviewers for their thoughtful reviews, and the authors for contributing their papers.

Publisher's Note Springer Nature remains neutral with regard to jurisdictional claims in published maps and institutional affiliations. 